\title{
Asymptotic Behaviors of Support Vector Machines with Gaussian Kernel
}

\section{S. Sathiya Keerthi}

mpessk@guppy.mpe.nus.edu.sg

Department of Mechanical Engineering, National University of Singapore,

Singapore 119260, Republic of Singapore

\section{Chih-Jen Lin}

cjlin@csie.ntu.edu.tw

Department of Computer Science and Information Engineering,

National Taiwan University, Taipei 106, Taiwan

Support vector machines (SVMs) with the gaussian (RBF) kernel have been popular for practical use. Model selection in this class of SVMs involves two hyperparameters: the penalty parameter $C$ and the kernel width $\sigma$. This letter analyzes the behavior of the SVM classifier when these hyperparameters take very small or very large values. Our results help in understanding the hyperparameter space that leads to an efficient heuristic method of searching for hyperparameter values with small generalization errors. The analysis also indicates that if complete model selection using the gaussian kernel has been conducted, there is no need to consider linear SVM.

\section{Introduction}

Given a training set of instance-label pairs $\left(x_{i}, y_{i}\right), i=1, \ldots, l$ where $x_{i} \in R^{n}$ and $y \in\{1,-1\}^{l}$, support vector machines (SVMs) (Vapnik, 1998) require the solution of the following (primal) optimization problem:

$$
\begin{array}{ll}
\min _{w, b, \xi} & \frac{1}{2} w^{T} w+C \sum_{i=1}^{l} \xi_{i} \\
\text { subject to } & y_{i}\left(w^{T} z_{i}+b\right) \geq 1-\xi_{i}, \\
& \xi_{i} \geq 0, i=1, \ldots, l .
\end{array}
$$

Here, training vectors $x_{i}$ are mapped into a higher- (maybe infinite-) dimensional space by the function $\phi$ as $z_{i}=\phi\left(x_{i}\right) . C>0$ is the penalty parameter of the error term. 
Usually we solve equation 1.1 by solving the following dual problem:

$$
\begin{array}{cl}
\min _{\alpha} & F(\alpha)=\frac{1}{2} \alpha^{T} Q \alpha-e^{T} \alpha \\
\text { subject to } & 0 \leq \alpha_{i} \leq C, i=1, \ldots, l, \\
& y^{T} \alpha=0
\end{array}
$$

where $e$ is the vector of all ones and $Q$ is an $l$ by $l$ positive semidefinite matrix. The $(i, j)$ th element of $Q$ is given by $Q_{i j} \equiv y_{i} y_{j} K\left(x_{i}, x_{j}\right)$, where $K\left(x_{i}, x_{j}\right) \equiv$ $\phi\left(x_{i}\right)^{T} \phi\left(x_{j}\right)$ is called the kernel function. Then $w=\sum_{i=1}^{l} \alpha_{i} y_{i} \phi\left(x_{i}\right)$ and

$$
\operatorname{sgn}\left(w^{T} \phi(x)+b\right)=\operatorname{sgn}\left(\sum_{i=1}^{l} \alpha_{i} y_{i} K\left(x_{i}, x\right)+b\right)
$$

is the decision function.

We are particularly interested in the gaussian kernel:

$$
K(\tilde{x}, \bar{x})=\exp \left(\frac{-\|\tilde{x}-\bar{x}\|^{2}}{2 \sigma^{2}}\right) .
$$

Our aim is to analyze the behaviors of the SVM classifier when $C$ and/or $\sigma^{2}$ take very small or very large values. The motivation is that such an analysis will help in understanding the hyperparameter space that will lead to efficient heuristic ways of searching for points in that space with small generalization errors. Some of the behaviors that we will discuss are known in the literature (although details associated with these are usually not written down carefully), but some key behaviors are new results that are not entirely obvious. Here is a summary of the asymptotic behaviors of the SVM classifier that are derived in this article:

- Severe underfitting (the entire data space is assigned to the majority class) occurs in the following cases: (1) $\sigma^{2}$ is fixed and $C \rightarrow 0,(2) \sigma^{2} \rightarrow 0$ and $C$ is fixed to a sufficiently small value, and (3) $\sigma^{2} \rightarrow \infty$ and $C$ is fixed.

- Severe overfitting (small regions around the training examples of the minority class are classified to be that class, while the rest of the data space is classified as the majority class) occurs in the case where $\sigma^{2} \rightarrow 0$ and $C$ is fixed to a sufficiently large value.

- If $\sigma^{2}$ is fixed and $C \rightarrow \infty$, the SVM classifier strictly separates the training examples of the two classes. This is a case of overfitting if the problem under consideration has noise.

- If $\sigma^{2} \rightarrow \infty$ and $C=\tilde{C} \sigma^{2}$ where $\tilde{C}$ is fixed, then the SVM classifier converges to the linear SVM classifier with penalty parameter $\tilde{C}$. 

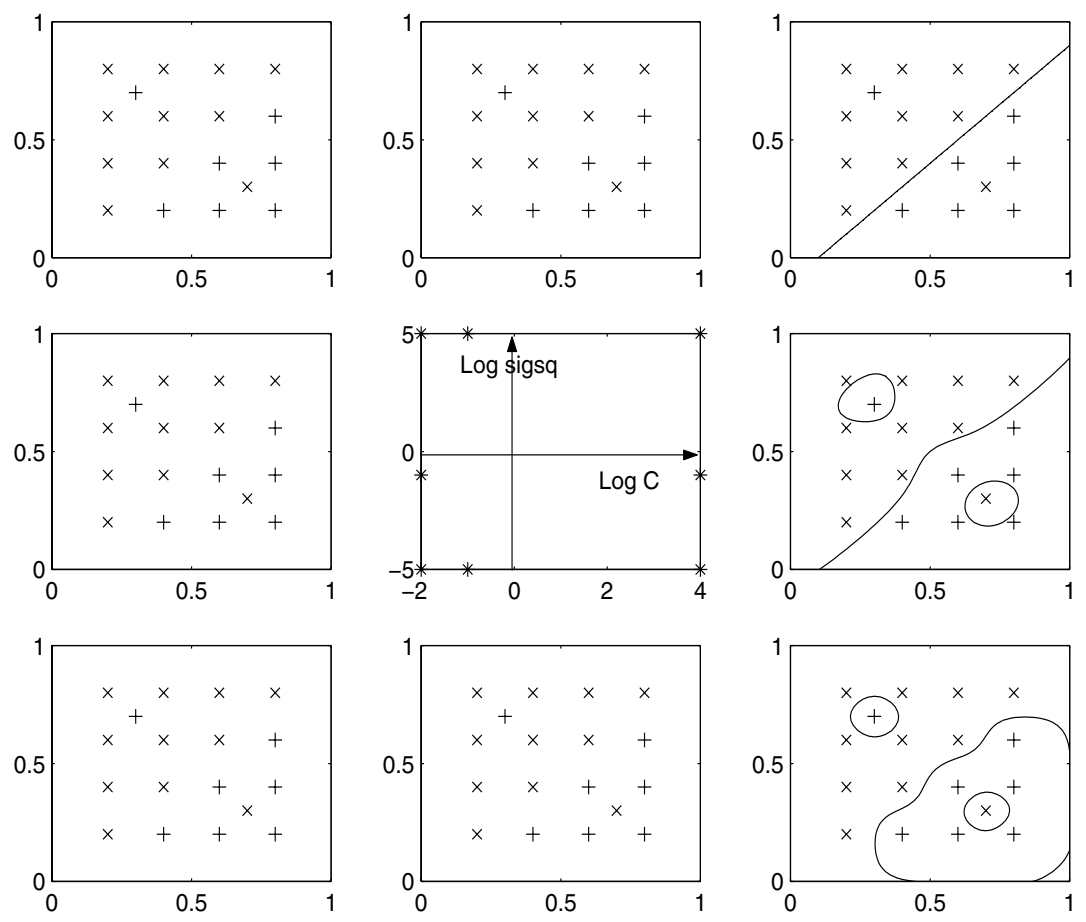

Figure 1: A figurative summary of the asymptotic behaviors. The problem has 11 examples in class $1(\times)$ and 7 examples in class $2(+)$. Thus, class 1 is the majority class, and class 2 is the minority class. The plot in the center shows the eight $\left(\log C, \log \sigma^{2}\right)$ pairs tried. The decision curves corresponding to these eight pairs are displayed in the surrounding plots at respective positions. Plots without a decision curve correspond to underfitting classifiers for which the entire input region is classified as class 1.

Figure 1 gives a summary of the asymptotic behaviors.

Asymptotic behaviors of the generalization error associated with the SVM classifier as $C$ and/or $\sigma^{2}$ take extreme values and can be understood by studying corresponding behaviors of the leave-one-out (LOO) error. The LOO error is computed as follows. For the $i$ th example, equations 1.1 and 1.2 are solved after leaving out that example. The resulting classifier is applied to check if the $i$ th example is misclassified. The procedure is repeated for each $i$. The fraction of misclassified examples is the LOO error.

This article is organized as follows. In section 2, we analyze the asymptotic behaviors of the SVM classifier using the gaussian kernel. The results lead to a simple and efficient heuristic model selection strategy described in section 3. Experiments show that the proposed method is competitive with 
the usual cross-validation search strategy in terms of generalization error achieved, while at the same time it is much more efficient.

\section{Asymptotic Behaviors}

To establish various asymptotic behaviors of the SVM decision function as well as the LOO error, we need the following assumption, which will be assumed throughout the article:

\section{Assumption 1:}

1. $l_{1}>l_{2}+1>2$ where $l_{1}$ and $l_{2}$ are the numbers of training examples in class 1 and class 2 , respectively. ${ }^{1}$

2. For $i \neq j, x_{i} \neq x_{j}$. That is, no two examples have identical $x$ vectors. $^{2}$

The following lemma is useful.

Lemma 1. For any given $\left(C, \sigma^{2}\right)$, the solution ( $\alpha$ ) of equation 1.2 is unique. Also, for every $\sigma^{2},\left\{z_{i} \mid y_{i}=1\right\}$ and $\left\{z_{i} \mid y_{i}=-1\right\}$ are linearly separable.

Proof. From Micchelli (1986), if the gaussian kernel is used and $x_{i} \neq x_{j} \forall i \neq$ $j$ from assumption $2, Q$ is positive definite. By corollary 1 of Chang and Lin (2001b), we get linear separability in $z$-space. Uniqueness of $\alpha$ follows from the fact that equation 1.2 is a strictly convex quadratic programming problem.

We now discuss the various asymptotic behaviors. As the results of each case are stated, it is useful to refer to the example shown in Figure 1. Wherever we come across results whose proofs do not shed any insight on the asymptotic behaviors, we only state the results and relegate the proofs to the appendix.

2.1 Case 1. $\sigma^{2}$ Fixed and $C \rightarrow 0$. It can be shown (see the proof of theorem 5 in Chang \& Lin, 2001b, for details) that if $C$ is smaller than a certain positive value, the following holds:

$$
\alpha_{i}=C \forall i \quad \text { with } y_{i}=-1 \text {. }
$$

\footnotetext{
${ }^{1}$ If $l_{2}>l_{1}+1>2$, then we can always interchange the two classes and apply all the results derived in this article. Cases where $\left|l_{1}-l_{2}\right| \leq 1$ or $\min \left\{l_{1}, l_{2}\right\}<2$ correspond to abnormal situations that are not worth discussing in detail since in practice, the number of examples in the two classes rarely satisfies any of these two conditions.

${ }^{2}$ This is a generic assumption that is easily satisfied if small, random perturbations are added to all training examples.
} 
Let us take one such $C$. Using equation 2.1 together with $\sum_{i=1}^{l} y_{i} \alpha_{i}=0$ and $l_{1}>l_{2}$, it is easy to see that there exists at least one $i$ for which $\alpha_{i}<C$ and $y_{i}=1$. For such an $i$, we have

$$
w^{T} z_{i}+b \geq 1
$$

For $C \rightarrow 0$, we have $\alpha_{i} \rightarrow 0$, and so $w^{T} z=\sum_{i=1}^{l} \alpha_{i} y_{i} K\left(x_{i}, x\right) \rightarrow 0$, where $z=\phi(x)$. These imply that if $X$ is any compact subset of $R^{n}$, then for any given $0<a<1$, there exists $\bar{C}>0$ such that for all $C \leq \bar{C}$, we have

$$
b \geq a \text { and }\left|\sum_{i=1}^{l} \alpha_{i} y_{i} K\left(x_{i}, x\right)\right| \leq \frac{a}{2} \forall x \in X .
$$

Hence, for all $C \leq \bar{C}$,

$$
f(x)>0 \forall x \in X
$$

In particular, if we take $X$ to be the compact subset of data space that is of interest to the given problem, then for sufficiently small $C$, every point in this subset is classified as class 1 .

The first part of assumption 1 allows us to use similar arguments for the case of equation 1.2 with one example left out. Then we can also show that as $C \rightarrow 0$, the number of LOO errors is $l_{2}$. Thus, $C \rightarrow 0$ corresponds to severe underfitting as expected. Furthermore, we have the following properties as $C \rightarrow 0$ :

1. $\|w\|^{2}=\alpha^{T} Q \alpha \rightarrow 0$.

2. $\lim _{C \rightarrow 0} \frac{1}{C} \sum_{i=1}^{l} \alpha_{i}=\lim _{C \rightarrow 0} \frac{2}{C} \sum_{i: y_{i}=-1} \alpha_{i}=2 l_{2}$.

3. Using the equality of primal and dual objective function values at optimality and the inequality $\alpha^{T} Q \alpha \leq l^{2} C^{2}$, we get

$$
\lim _{C \rightarrow 0} \sum_{i=1}^{l} \xi_{i}=\lim _{C \rightarrow 0} \frac{1}{C}\left(\sum_{i=1}^{l} \alpha_{i}-\alpha^{T} Q \alpha\right)=2 l_{2} .
$$

It is useful to interpret the above asymptotic results geometrically; in particular, study the movement of the top, middle, and bottom planes defined by $w^{T} z+b=1, w^{T} z+b=0$, and $w^{T} z+b=-1$ as $C \rightarrow 0$. By equation 2.2, at least one example of class 1 lies on or above the top plane. By property 1 given above, the distance between the top and bottom planes (which equals $2 /\|w\|)$ goes to infinity. Hence, the middle and bottom planes are forced to move down farther and farther away from the location where the training points are located, causing the half-space defined by $w^{T} z+b \geq 0$ to cover $X$ entirely, the compact subset of interest to the problem, after $C$ becomes sufficiently small. 
Remark 1. The results given above for $C \rightarrow 0$ are general and apply to nongaussian kernels also, assuming, of course, that all hyperparameters associated with the kernel function are kept fixed. The results also apply if $Q$ is a bounded function of $C$ since theorem 5 of Chang and Lin (2001b) holds for this case.

Remark 2. For kernels whose values are bounded (e.g., the gaussian kernel), there is $\bar{C}$ such that equation 2.3 holds for all $x \in R^{n}$. Thus, for all $C \leq \bar{C}$,

$$
f(x)>0 \forall x \in R^{n} .
$$

That is, for all $C \leq \bar{C}$, every point is classified as class 1 .

2.2 Case 2. $\sigma^{2}$ Fixed and $C \rightarrow \infty$. By lemma $1,\left\{z_{i} \mid y_{i}=1\right\}$ and $\left\{z_{i} \mid y_{i}=\right.$ $-1\}$ are linearly separable. This implies that it is possible to set $\xi_{i}=0 \forall i$ while still remaining feasible for equation 1.1. Thus, as $C \rightarrow \infty$, the solution of equation 1.1 approaches the solution of the hard margin problem:

$$
\begin{aligned}
\min _{w, b} & \frac{1}{2} w^{T} w \\
\text { subject to } & y_{i}\left(w^{T} z_{i}+b\right) \geq 1, i=1, \ldots, l .
\end{aligned}
$$

A formal treatment of this is in Lin (2001), which shows that if equation 2.4 is feasible, there exists a $C^{*}$ such that for $C \geq C^{*}$, the solution set of equation 1.1 is the same as that of equation 2.4. An easy way to see this result is to solve equation 1.2 with $C=\infty$, obtain the $\left\{\alpha_{i}\right\}$, and set $C^{*}=\max _{i} \alpha_{i}$.

The limiting SVM classifier classifies all training examples correctly, and so it is an overfitting classifier. In particular, severe overfitting occurs when $\sigma^{2}$ is small since the flexibility of the classifier is high when $\sigma^{2}$ is small.

For the case of $C \rightarrow \infty$, it is not possible to make any conclusions about the actual value of the LOO error. That value depends on the data set as well as on the value of $\sigma^{2}$. However, after equation 1.2 is solved using all the examples, it is possible to give bounds on the LOO error (Joachims, 2000; Vapnik \& Chapelle, 2000) without solving the quadratic programs obtained by leaving out one example at a time.

2.3 Case 3. $C$ Is Fixed and $\sigma^{2} \rightarrow 0$. Let us define $\delta_{i j}=1$ for $i=j$ and $\delta_{i j}=0$ if $i \neq j$. Since $e^{-\left\|x_{i}-x_{j}\right\|^{2} /\left(2 \sigma^{2}\right)} \rightarrow \delta_{i j}$ as $\sigma^{2} \rightarrow 0$, we consider the following problem:

$$
\begin{array}{cl}
\min _{\alpha} & \frac{1}{2} \alpha^{T} \alpha-e^{T} \alpha \\
\text { subject to } & 0 \leq \alpha_{i} \leq C, i=1, \ldots, l, \\
& y^{T} \alpha=0 .
\end{array}
$$


Using lemma 2 (the proof is in section A.1), as $\sigma^{2} \rightarrow 0$, the solution of equation 1.2 converges to that of equation 2.5. Since $l_{1}>l_{2}$, the solution of equation 1.2 has $0<\alpha_{i}<C$ for at least one $i .^{3}$ Thus, $b$ is uniquely determined, and as $\sigma^{2} \rightarrow 0$, it approaches the value of $b$ corresponding to the primal form of equation 2.5 .

Therefore, let us study the solution of equation 2.5. In section A.2, we show that its solution is given by $\alpha_{i}=\alpha^{+}$if $y_{i}=1$ and $\alpha_{i}=\alpha^{-}$if $y_{i}=-1$, where

$$
\alpha^{-}=\left\{\begin{array}{ll}
C_{\lim } & \text { if } C \geq C_{\lim } \\
C & \text { if } C<C_{\lim },
\end{array} \quad \alpha^{+}= \begin{cases}2 l_{2} / l & \text { if } C \geq C_{\lim } \\
l_{2} C / l_{1} & \text { if } C<C_{\lim }\end{cases}\right.
$$

and $C_{\lim }=2 l_{1} / l$. The threshold parameter $b$ in the primal form corresponding to equation 2.5 can be determined using the fact that $0<\alpha^{+}<C$ (and hence all class 1 examples lie on the top plane defined by $w^{T} z+b=1$ ):

$$
b= \begin{cases}\left(l_{1}-l_{2}\right) / l & \text { if } C \geq C_{\lim }, \\ 1-l_{2} C / l_{1} & \text { if } C<C_{\lim } .\end{cases}
$$

Consider the classifier function $f(x)=w^{T} z+b$ corresponding to equation 2.5. In section A.2, we also show the following:

1. If $C \geq C_{\lim } / 2, f$ classifies all training examples correctly and classifies the rest of the space as class 1 . Thus, it overfits the training data.

2. If $C<C_{\lim } / 2$, then $f$ classifies the entire space as class 1 , and so it underfits the training data.

3. The number of LOO errors is $l_{2}$.

Consider the SVM classifier corresponding to the gaussian kernel for small values of $\sigma^{2}$. Even though the number of LOO errors tends to $l_{2}$ for all $C$, it is important to note that the SVM classifier is qualitatively very different for large $C$ and small $C$. For large $C$, there are small regions around each example of class 2 that are classified as class 2 (overfitting), while for small $C$, there are no such regions (underfitting).

It is interesting to note that if $\sigma^{2}$ is small and $C$ is greater than a threshold that is around $C_{\text {lim }}$, from equation 2.6, the SVM classifier does not depend on $C$. Thus, contour lines of constant generalization error are parallel to the $C$ axis in the region where $\sigma^{2}$ is small and $C$ is large.

\footnotetext{
${ }^{3}$ As we show below (see equation 2.6), the solution of equation 2.5 is well in the interior of $(0, C)$ for at least one $i$. Since for small values of $\sigma^{2}$, the solution of equation 1.2 approaches that of equation 2.5, it follows that the solution of equation 1.2 also has $0<$ $\alpha_{i}<C$ for at least one $i$.
} 
2.4 Case 4. $C$ Is Fixed and $\sigma^{2} \rightarrow \infty$. When $\sigma^{2} \rightarrow \infty$, we can write

$$
\begin{aligned}
K(\tilde{x}, \bar{x}) & =\exp \left(-\|\tilde{x}-\bar{x}\|^{2} / 2 \sigma^{2}\right) \\
& =1-\frac{\|\tilde{x}-\bar{x}\|^{2}}{2 \sigma^{2}}+o\left(\|\tilde{x}-\bar{x}\|^{2} / \sigma^{2}\right) \\
& =1-\frac{\|\tilde{x}\|^{2}}{2 \sigma^{2}}-\frac{\|\bar{x}\|^{2}}{2 \sigma^{2}}+\frac{\tilde{x}^{T} \bar{x}}{\sigma^{2}}+o\left(\|\tilde{x}-\bar{x}\|^{2} / \sigma^{2}\right) .
\end{aligned}
$$

Now consider equation 1.2. Using the simplification given above, we can write the first (quadratic) term of the objective function in equation 1.2 as

$$
\sum_{i} \sum_{j} \alpha_{i} \alpha_{j} y_{i} y_{j} K\left(x_{i}, x_{j}\right)=T_{1}+\frac{T_{2}+T_{3}+T_{4}}{2 \sigma^{2}}+\frac{1}{2} \sum_{i} \sum_{j} \alpha_{i} \alpha_{j} y_{i} y_{j} \frac{\Delta_{i j}}{\sigma^{2}},
$$

where

$$
\begin{aligned}
& T_{1}=\sum_{i} \sum_{j} \alpha_{i} \alpha_{j} y_{i} y_{j}, \quad T_{2}=-\sum_{i} \sum_{j} \alpha_{i} \alpha_{j} y_{i} y_{j}\left\|x_{i}\right\|^{2} \\
& T_{3}=-\sum_{i} \sum_{j} \alpha_{i} \alpha_{j} y_{i} y_{j}\left\|x_{j}\right\|^{2}, \quad T_{4}=2 \sum_{i} \sum_{j} \alpha_{i} \alpha_{j} y_{i} y_{j} x_{i}^{T} x_{j}, \quad \text { and } \\
& \lim _{\sigma^{2} \rightarrow \infty} \Delta_{i j}=0 .
\end{aligned}
$$

By the equality constraint of equation 1.2, $T_{1}=\left(\sum_{i} \alpha_{i} y_{i}\right)^{2}=0$. We can also rewrite $T_{2}$ as $T_{2}=\left(\sum_{i} \alpha_{i} y_{i}\left\|x_{i}\right\|^{2}\right)\left(\sum_{j} \alpha_{j} y_{j}\right)=0$. In a similar way, $T_{3}=0$. By defining

$$
\tilde{\alpha}_{i}=\frac{\alpha_{i}}{\sigma^{2}} \forall i
$$

equation 1.2 can be written as ${ }^{4}$

$$
\begin{aligned}
\min _{\tilde{\alpha}} & \frac{F}{\sigma^{2}}=\frac{1}{2} \sum_{i} \sum_{j} \tilde{\alpha}_{i} \tilde{\alpha}_{j} y_{i} y_{j} \tilde{K}_{i j}-\sum_{i} \tilde{\alpha}_{i} \\
\text { subject to } & 0 \leq \tilde{\alpha}_{i} \leq \tilde{C}, i=1, \ldots, l, \\
& y^{T} \tilde{\alpha}=0
\end{aligned}
$$

where $\tilde{K}_{i j}=x_{i}^{T} x_{j}+\Delta_{i j}$ and

$$
\tilde{C}=\frac{C}{\sigma^{2}} \text {. }
$$

\footnotetext{
${ }^{4}$ To do this, note that we need to divide the objective function by the term $\sigma^{2}$.
} 
Remark 3. Note that $\tilde{K}_{i j}$ may not correspond to a valid kernel satisfying the Mercer's condition. But that is immaterial since we always operate with the constraint $y^{T} \tilde{\alpha}=0$. In the presence of this constraint, equations 1.2 and 2.11 are equivalent.

Remark 4. If $C$ is fixed at some value and $\sigma^{2}$ is made large, $\tilde{C}$ of equation 2.11 goes to zero, and so the situation is similar to case 1 , discussed at the beginning of this section. By equation $2.9, \tilde{K}_{i j}$ is a bounded function for large $\sigma^{2}$ (or, equivalently, for small $\tilde{C}$ ). By the last sentence of remark 1 , results of case 1 can be applied here. Thus, for $C$ fixed and $\sigma^{2} \rightarrow \infty$, equation 2.11 corresponds to a severely underfitting classifier. Since equations 2.11 and 1.2 correspond to the same problem in different forms, they have the same primal decision function (for full details, see equation A.8). Therefore, in this situation, we get a severely underfitting classifier.

For a given $\tilde{C}$, as $\sigma^{2} \rightarrow \infty$ and $C$ varies with $\sigma^{2}$ as given by equation 2.12, we can see that equation 2.11 is close to the following linear SVM problem:

$$
\begin{aligned}
\min _{\tilde{\alpha}} & \frac{1}{2} \sum_{i} \sum_{j} \tilde{\alpha}_{i} \tilde{\alpha}_{j} y_{i} y_{j} x_{i}^{T} x_{j}-\sum_{i} \tilde{\alpha}_{i} \\
\text { subject to } \quad & 0 \leq \tilde{\alpha}_{i} \leq \tilde{C}, i=1, \ldots, l, \\
& y^{T} \tilde{\alpha}=0 .
\end{aligned}
$$

We are interested in their corresponding decision functions, which can lead us to analyze the performance of equation 1.1. Now the primal form of equation 2.13 is

$$
\begin{array}{ll}
\min _{\tilde{w}, \tilde{b}, \tilde{\xi}} & \frac{1}{2} \tilde{w}^{T} \tilde{w}+\tilde{C} \sum_{i=1}^{l} \tilde{\xi}_{i} \\
\text { subject to } & y_{i}\left(\tilde{w}^{T} x_{i}+\tilde{b}\right) \geq 1-\tilde{\xi}_{i}, \\
& \tilde{\xi}_{i} \geq 0, i=1, \ldots, l .
\end{array}
$$

Let $\left(w\left(\sigma^{2}\right), b\left(\sigma^{2}\right)\right)$, and $(\tilde{w}, \tilde{b})$ denote primal optimal solutions of equations 1.1 and 2.14, respectively. We then have the following theorem:

Theorem 1. For any $x, \lim _{\sigma^{2} \rightarrow \infty} w\left(\sigma^{2}\right)^{T} z=\tilde{w}^{T} x$. If the optimal $\tilde{b}$ of equation 2.14 is unique, then $\lim _{\sigma^{2} \rightarrow \infty} b\left(\sigma^{2}\right)=\tilde{b}$ and hence the following also hold:

1. For any $x$,

$$
\lim _{\sigma^{2} \rightarrow \infty} w\left(\sigma^{2}\right)^{T} z+b\left(\sigma^{2}\right)=\tilde{w}^{T} x+\tilde{b} .
$$


2. If $\tilde{w}^{T} x+\tilde{b} \neq 0$, then for $\sigma^{2}$ sufficiently large,

$$
\operatorname{sgn}\left(w\left(\sigma^{2}\right)^{T} z+b\left(\sigma^{2}\right)\right)=\operatorname{sgn}\left(\tilde{w}^{T} x+\tilde{b}\right) .
$$

The proof is in section A.3. Thus, for a given $\tilde{C}$, the limiting SVM gaussian kernel classifier as $\sigma^{2} \rightarrow \infty$ is the same as the SVM linear kernel classifier for $\tilde{C}$. Hereafter, we will simply refer to the SVM linear kernel classifier as linear SVM. The above analysis can also be extended to show that as $\sigma^{2} \rightarrow \infty$, the LOO error corresponding to equations 1.1 and 2.13 is the same.

The above results also show that in the part of the hyperparameter space where $\sigma^{2}$ is large, if $\left(C_{1}, \sigma_{1}^{2}\right)$ and $\left(C_{2}, \sigma_{2}^{2}\right)$ are related by $C_{1} / \sigma_{1}^{2}=C_{2} / \sigma_{2}^{2}=\tilde{C}$, the classifiers corresponding to the two combinations are nearly the same. Hence, both will give nearly the same value for generalization error (or an estimate of it, such as $k$-fold cross-validation error or LOO error). Thus, in this part of the hyperparameter space, contour lines of such functions will be straight lines with slope $1: \log \sigma^{2}=\log C-\log \tilde{C}$. Then all classifiers defined by points on that straight line for large $\sigma^{2}$ are nearly the same as the linear SVM classifier corresponding to $\tilde{C}$.

Given that for any $x, \lim _{\sigma^{2} \rightarrow \infty} w\left(\sigma^{2}\right)^{T} z=\tilde{w}^{T} x$ holds without any assumption, the assumption on the uniqueness of $\tilde{b}$ in theorem 1 should be viewed as only a minor technical irritant. ${ }^{5}$ For normal situations, the uniqueness assumption is a reasonable one to make. Unless $\tilde{C}$ is very small, typically there will be at least one $\tilde{\alpha}_{i}$ strictly in between 0 and $\tilde{C}$; when such an $\tilde{\alpha}_{i}$ exists, lemma 3 in section A.1 (as applied to section 2.14) implies the uniqueness of $\tilde{b}$. The case of a very small $\tilde{C}$ corresponds to the upper left part of the plane in which $\log C$ and $\log \sigma^{2}$ are the horizontal and vertical axes. We can easily see this by considering $C$ fixed and increasing $\sigma^{2}$ to large values (the upper part) or considering $\sigma^{2}$ fixed and decreasing $C$ to small values (the left part). As remark 4 and case 1 of this section show, each of these asymptotic behaviors corresponds to a severely underfitting SVM decision function.

Finally, theorem 1 also indicates that if complete model selection on $\left(C, \sigma^{2}\right)$ using the gaussian kernel has been conducted, there is no need to consider linear SVM. This helps in the selection of kernels.

\section{A Method of Model Selection}

It is usual to take $\log C$ and $\log \sigma^{2}$ as the parameters of the hyperparameter space. Putting together the results derived in the previous section, it is easy to see that in the asymptotic (outer) regions of the $\left(\log C, \log \sigma^{2}\right)$

${ }^{5}$ The assumption is needed to state results cleanly. If $\tilde{b}$ is nonunique, SVM classifiers also become nonunique, and then it becomes clumsy to talk about convergence of SVM decision functions. 


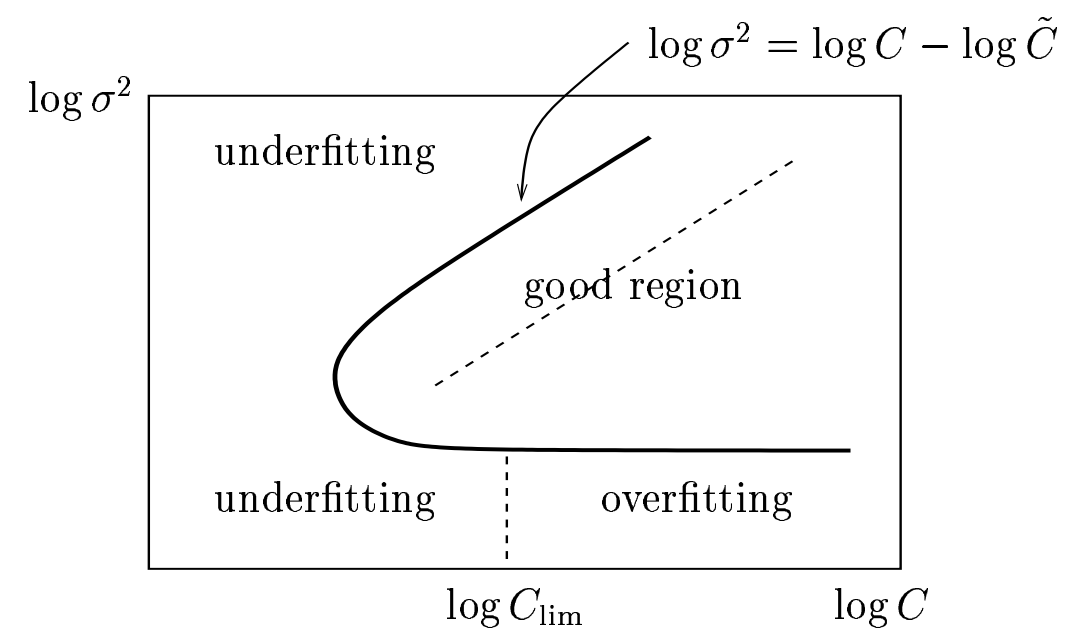

Figure 2: A rough boundary curve separating the underfitting/overfitting region from the "good" region. For each fixed $\tilde{C}$, the equation $\log \sigma^{2}=\log C-\log \tilde{C}$ defines a straight line of unit slope. As $\sigma^{2} \rightarrow \infty$ along this line, the SVM classifier converges to the linear SVM classifier with penalty parameter $\tilde{C}$. The dotted line corresponds to the choice of $\tilde{C}$ that gives the optimal generalization error for the linear SVM.

space, there exists a contour of generalization error (or an estimate such as LOO error or $k$-fold cross validation error) that looks like that shown in Figure 2 and helps separate the hyperparameter space into two regions: an overfitting/underfitting region and a good region (which most likely has the hyperparameter set with the best generalization error). (For LOO, recall that in the underfitting/overfitting region, the number of $\mathrm{LOO}$ errors is $l_{2}$.) The straight line with unit slope in the large $\sigma^{2}$ region $\left(\log \sigma^{2}=\log C-\log \tilde{C}\right)$ corresponds to the choice of $\tilde{C}$, which is small enough to make the linear SVM an underfitting one. The presence of a separating contour as outlined in Figure 2 has been observed on a number of real-world data sets (Lee, 2001).

When searching for a good set of values for $\log C$ and $\log \sigma^{2}$, it is usual to form a two-dimensional uniform grid (say $r \times r$ ) of points in this space and find a combination that gives the least value for some estimate of generalization error. This is expensive since it requires trying $r^{2}\left(C, \sigma^{2}\right)$ pairs. The earlier discussion relating to Figure 2 suggests a simple and efficient heuristic method for finding a hyperparameter set with small generalization error: form a line of unit slope that cuts through the middle part of the good region (see the dashed line in Figure 2) and search on it for a good set of hyperparameters. The $\tilde{C}$ that defines this line can be set to the optimal value 
of penalty parameter for the linear SVM. Thus, we propose the following procedure:

1. Search for the best $C$ of linear SVM and call it $\tilde{C}$.

2. Fix $\tilde{C}$ from step 1 , and search for the best $\left(C, \sigma^{2}\right)$ satisfying $\log \sigma^{2}=$ $\log C-\log \tilde{C}$ using the gaussian kernel.

The idea is that as $\sigma^{2} \rightarrow \infty$, SVM with gaussian kernel behaves like linear SVM, and so the best $\tilde{C}$ should happen in the upper part of the "good" region in Figure 2. Then a search on the line defined by $\log \sigma^{2}=\log C-\log \tilde{C}$ gives an even better point in the "good" region. In many practical pattern recognition problems, a linear classifier already gives a reasonably good performance, and some added nonlinearities help obtain finer improvements in accuracy. Step 2 of our procedure can be thought of as a simple way of injecting the required nonlinearities via the gaussian kernel. Since the procedure involves only two one-dimensional searches, it requires only $2 r$ pairs of $\left(C, \sigma^{2}\right)$ to be tried.

To test the goodness of the proposed method, we compare it with the usual method of using a two-dimensional grid search. For both, fivefold cross-validation was used to obtain estimates of generalization error. For the usual method, we uniformly discretize the $[-10,10] \times[-10,10]$ region to $21^{2}=441$ points. At each point, a fivefold cross-validation is conducted. The point with the best $\mathrm{CV}$ accuracy is chosen and used to predict the test data.

For the proposed method, we search for $\tilde{C}$ by fivefold cross-validation on linear SVM using uniformly spaced $\log C$ values in $[-8,2]$. Then we discretize $[-8,8]$ as values of $\log \sigma^{2}$ and check all points satisfying $\log \sigma^{2}=$ $\log C-\log \tilde{C}$. Because now fewer points have to be tried, we use the smaller grid spacing of 0.5 for both discretizations. The total number of points tried is 54 .

To evaluate empirically the usefulness of the proposed method, we consider several binary problems from Rätsch (1999). For each problem, Rätsch (1999) gives 100 realizations of the given data set into (training set, test set) partitions. We consider only the first of those realizations. In addition, the problem adult, from the UCI "adult" data set (Blake \& Merz, 1998), and the problem web, both as compiled by Platt (1998), are also included. For each of these two data sets, there are several realizations. For our study here, we consider only the realization with the smallest training set; the full data set with training data (including duplicated ones) removed is taken as the test set. For all data sets used, Table 1 gives the number of input variables, the number of training examples, and the number of test examples. All data sets are directly used as given in the mentioned references, without any further normalization or scaling.

The SVM software LIBSVM (Chang \& Lin, 2001a), which implements a decomposition method, is employed for solving equation 1.2. Table 1 presents 
Table 1: Comparison of the Model Selection Methods.

\begin{tabular}{lrrrrr}
\hline Problem & $\begin{array}{r}\text { Number of } \\
\text { Inputs }\end{array}$ & $\begin{array}{r}\text { Number of } \\
\text { Training } \\
\text { Examples }\end{array}$ & $\begin{array}{r}\text { Number of } \\
\text { Test } \\
\text { Examples }\end{array}$ & $\begin{array}{r}\text { Test Set Error of } \\
\text { Usual Grid } \\
\text { Method }\end{array}$ & $\begin{array}{r}\text { Test Set Error of } \\
\text { Proposed } \\
\text { Method }\end{array}$ \\
\hline banana & 2 & 400 & 4900 & $0.1235(6,-0)$ & $0.1178(-2,-2)$ \\
diabetes & 8 & 468 & 300 & $0.2433(4,7)$ & $0.2433(4,6)$ \\
image & 18 & 1300 & 1010 & $0.02475(9,4)$ & $0.02475(1,0.5)$ \\
splice & 60 & 1000 & 2175 & $0.09701(1,4)$ & $0.1011(0,4)$ \\
ringnorm & 20 & 400 & 7000 & $0.01429(-2,2)$ & $0.018(-3,2)$ \\
twonorm & 20 & 400 & 7000 & $0.031(1,3)$ & $0.02914(1,4)$ \\
waveform & 21 & 400 & 4600 & $0.1078(0,3)$ & $0.1078(0,3)$ \\
tree & 18 & 700 & 11,692 & $0.1132(8,4)$ & $0.1246(2,2)$ \\
adult & 123 & 1605 & 29,589 & $0.1614(5,6)$ & $0.1614(5,6)$ \\
web & 300 & 2477 & 38,994 & $0.02223(5,5)$ & $0.02223(5,5)$ \\
\hline
\end{tabular}

Note: For each approach, apart from the test error, the optimal $\left(\log C, \log \sigma^{2}\right)$ pair is also given.

the test error of the two methods, as well as the corresponding chosen values of $\log C$ and $\log \sigma^{2}$. It can be clearly seen that the new method is very competitive with the usual method in terms of test set accuracy. For large data sets, the proposed method has the great advantage that it checks many fewer points on the $\left(\log C, \log \sigma^{2}\right)$ plane, and so the savings in computing time can be large.

Note that in the chosen problems, the following quantities have a reasonably wide range: test error $(1.5 \%$ to $25 \%)$, the number of input variables (2 to 300), and the number of training examples (400 to 2477), and so the empirical evaluation demonstrates the applicability of the proposed approach to different types of data sets.

A remaining issue is how to decide the range of $\log C$ for determining $\tilde{C}$ in step 1 . From Table 1 , we can see that $\log \tilde{C}=\log C-\log \sigma^{2}$ is usually not a large number. Furthermore, we observe that for all problems, after $C$ is greater than a certain threshold, the cross-validation accuracy of the linear SVM is about the same. Therefore, if we start searching from small C values and go on to large $C$ values, the search can be stopped after the $C V$ accuracy stops varying much. An example of the variation of the fivefold CV accuracy of linear SVM is given in Figure 3.

For linear SVMs, we can formally establish that there exists a finite limiting value $C^{*}$ such that for $C \geq C^{*}$, the solution of the linear SVM remains unchanged. If $\left\{x_{i}: y_{i}=1\right\}$ and $\left\{x_{i}: y_{i}=-1\right\}$ are linearly separable, then the above result is easy to appreciate; the same ideas used in case 2 can be applied to show this. However, if $\left\{x_{i}: y_{i}=1\right\}$ and $\left\{x_{i}: y_{i}=-1\right\}$ are not linearly separable (which is typically the case), the result is nontrivial to 


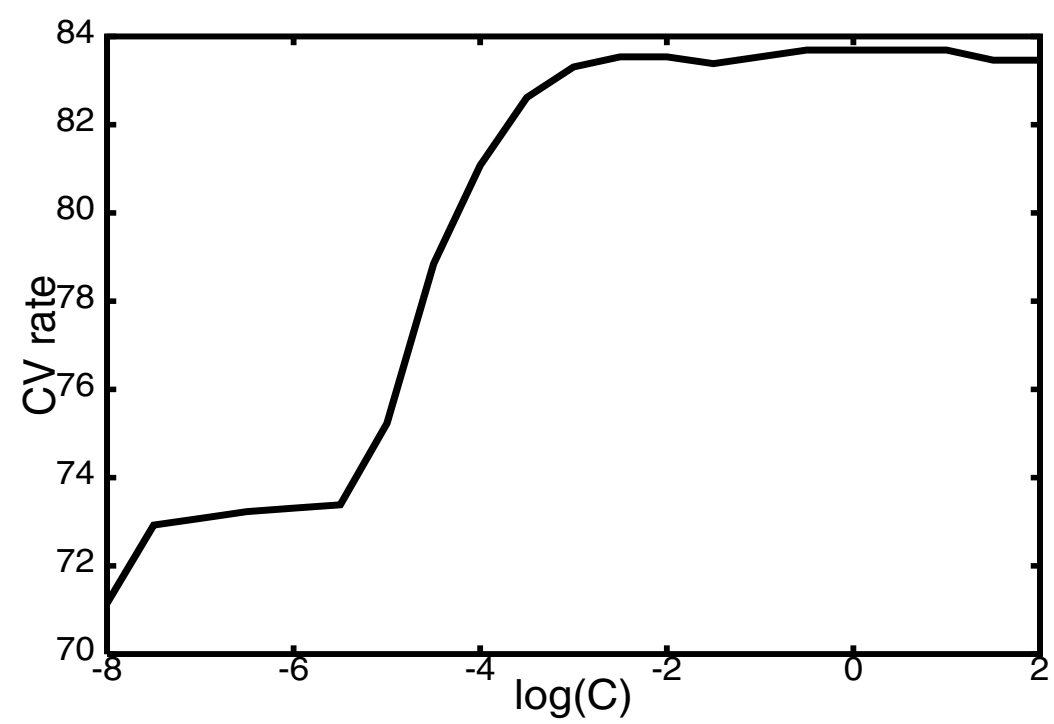

Figure 3: Variation of CV accuracy of linear SVM with $C$ for the image problem.

establish. Here, we prove the following theorem:

Theorem 2. There exists a finite value $C^{*}$ and $\left(w^{*}, b^{*}\right)$ such that $(w, b)=$ $\left(w^{*}, b^{*}\right)$ solves equation $1.2, \forall C \geq C^{*}$. If this decision function is used, the LOO error is same for all $C \geq C^{*}$. Moreover, this $w^{*}$ is unique.

Details of the proof are in section A.4.

\section{Appendix}

\section{A.1 Two Useful Lemmas.}

Lemma 2. Consider an optimization problem with the form 1.2 and $Q$ is a function of $\sigma^{2}$ (denoted as $Q\left(\sigma^{2}\right)$ ). Let $\alpha\left(\sigma^{2}\right)$ be its solutions. For a given number a, if

$$
Q^{*} \equiv \lim _{\sigma^{2} \rightarrow a} Q\left(\sigma^{2}\right)
$$

exists, then there exists a convergent sequence $\left\{\alpha\left(\sigma_{k}^{2}\right)\right\}$ with $\sigma_{k}^{2} \rightarrow a$, and the limit of any such sequence is an optimal solution of equation 1.2 with the Hessian matrix $Q^{*}$. Moreover, if $Q^{*}$ is positive definite, $\lim _{\sigma^{2} \rightarrow a} \alpha\left(\sigma^{2}\right)$ exists. 
Proof. The feasible region of equation 1.2 is independent of $\sigma^{2}$ so is compact. Then there exists a convergent sequence $\left\{\alpha\left(\sigma_{k}^{2}\right)\right\}$ with $\lim _{k \rightarrow \infty} \sigma_{k}^{2}=a$. For any one such sequence, we have

$$
\begin{aligned}
& \frac{1}{2} \alpha\left(\sigma_{k}^{2}\right)^{T} Q\left(\sigma_{k}^{2}\right) \alpha\left(\sigma_{k}^{2}\right)-e^{T} \alpha\left(\sigma_{k}^{2}\right) \leq \frac{1}{2}\left(\alpha^{*}\right)^{T} Q\left(\sigma_{k}^{2}\right) \alpha^{*}-e^{T} \alpha^{*}, \quad \text { and } \\
& \frac{1}{2}\left(\alpha^{*}\right)^{T} Q^{*} \alpha^{*}-e^{T} \alpha^{*} \leq \frac{1}{2} \alpha\left(\sigma_{k}^{2}\right)^{T} Q^{*}\left(\sigma_{k}^{2}\right) \alpha\left(\sigma_{k}^{2}\right)-e^{T} \alpha\left(\sigma_{k}^{2}\right),
\end{aligned}
$$

where $\alpha^{*}$ is any optimal solution of equation 1.2 with the Hessian matrix $Q^{*}$. If $\alpha\left(\sigma_{k}^{2}\right)$ goes to $\bar{\alpha}$, taking the limit of equation A.1,

$$
\frac{1}{2}\left(\alpha^{*}\right)^{T} Q^{*} \alpha^{*}-e^{T} \alpha^{*}=\frac{1}{2} \bar{\alpha}^{T} Q^{*} \bar{\alpha}-e^{T} \bar{\alpha} .
$$

Thus, $\bar{\alpha}$ is an optimal solution too.

If $Q^{*}$ is positive definite, equation 1.2 is a strictly convex problem with a unique optimal solution. This implies that $\lim _{\sigma^{2} \rightarrow a} \alpha\left(\sigma^{2}\right)$ exists.

Lemma 3. If equation 1.2 has an optimal solution with at least one free variable (i.e., $0<\alpha_{i}<C$ for at least one $i$ ), then the optimal b of equation 1.1 is unique.

Proof. The Karush-Kuhn-Tucker (KKT) condition (i.e. the optimality condition) of equation 1.2 is that if $\alpha$ is an optimal solution, there are a number $b$ and two nonnegative vectors $\lambda$ and $\mu$ such that

$$
\begin{aligned}
& \nabla F(\alpha)+b y=\lambda-\mu, \\
& \lambda_{i} \alpha_{i}=0, \quad \mu_{i}(C-\alpha)_{i}=0, \quad \lambda_{i} \geq 0, \mu_{i} \geq 0, \quad i=1, \ldots, l,
\end{aligned}
$$

where $\nabla F(\alpha)=Q \alpha-e$ is the gradient of $F(\alpha)=1 / 2 \alpha^{T} Q \alpha-e^{T} \alpha$. This can be rewritten as

$$
\begin{array}{ll}
\nabla F(\alpha)_{i}+b y_{i} \geq 0 & \text { if } \alpha_{i}=0, \\
\nabla F(\alpha)_{i}+b y_{i} \leq 0 & \text { if } \alpha_{i}=C, \\
\nabla F(\alpha)_{i}+b y_{i}=0 & \text { if } 0<\alpha_{i}<C .
\end{array}
$$

Note that

$$
\nabla F(\alpha)_{i}=y_{i} w^{T} z_{i}-1
$$

is independent of different optimal solutions $\alpha$ as the primal optimal solution $w$ is unique.

Let $(w, b, \xi)$ denote a primal solution. As already said, $w$ is unique. By convexity of the solution set, the set of all possible $b$ solutions, $B$ is an interval. 
Once $b$ is chosen, $\xi$ is uniquely defined. By assumption, there exists $b \in B$ and a corresponding Lagrange multiplier vector $\alpha(b)$ with a free alpha, say, $0<\alpha(b)_{k}<C$. Thus, $\alpha(b)$ is an optimal solution of equation 1.2 and so, by equation A.2, $\nabla F(\alpha)_{k}+b y_{k}=0$. Denote

$$
\begin{aligned}
& A_{0}=\left\{i \mid \nabla F(\alpha)_{i}+b y_{i}>0\right\}, \quad A_{C}=\left\{i \mid \nabla F(\alpha)_{i}+b y_{i}<0\right\}, \quad \text { and } \\
& A_{F}=\left\{i \mid i \notin A_{0} \cup A_{C}\right\} .
\end{aligned}
$$

Let us define $2 e$ and $2 f$ to be the minimum and maximum of the following set:

$$
\begin{aligned}
\left\{b^{\text {new }} \mid \nabla F(\alpha)_{i}+b^{\text {new }} y_{i}\right. & >0 \text { if } i \in A_{0} ; \\
\nabla F(\alpha)_{i}+b^{\text {new }} y_{i} & \left.<0 \text { if } i \in A_{C}\right\} .
\end{aligned}
$$

Clearly $e<b<f$. Suppose $B$ is not a singleton. Now choose $b^{\text {new }} \in B \cap[e, f]$ such that $b^{\text {new }} \neq b$. Let $\alpha\left(b^{\text {new }}\right)$ be any Lagrange multiplier corresponding to $b^{\text {new }}$. Thus, $\alpha\left(b^{\text {new }}\right)$ and $b^{\text {new }}$ satisfy equation A.2. Suppose $b^{\text {new }}>b$ and $y_{k}=1$. Then

$$
\nabla F(\alpha)_{k}+b^{\text {new }} y_{k}>0 \text { so } \alpha\left(b^{\text {new }}\right)_{k}=0<\alpha(b)_{k}
$$

If we use equation A.2 as applied to $(b, \alpha(b))$ and $\left(b^{\text {new }}, \alpha\left(b^{\text {new }}\right)\right)$, equation A.3 implies the following: $\alpha(b)_{i}=\alpha\left(b^{\text {new }}\right)_{i} \forall i \in A_{0} \cup A_{C}$ with $y_{i}=y_{k}$; also, $\alpha(b)_{i} \geq \alpha\left(b^{\text {new }}\right)_{i} \forall i \in A_{F}$ with $y_{i}=y_{k}$. Note that $k$ is an element of this second group. Thus, with equation A.4,

$$
\sum_{i: y_{i}=y_{k}} \alpha(b)_{i}>\sum_{i: y_{i}=y_{k}} \alpha\left(b^{\text {new }}\right)_{i} .
$$

This is a violation of the fact that both $\alpha(b)$ and $\alpha\left(b^{\text {new }}\right)$ are solutions of equation 1.2 since, for a given dual solution $\alpha$, the dual cost is $\left(\|w\|^{2} / 2\right)-$ $2 \sum_{i: y_{i}=1} \alpha_{i}$ and the first term is the same for $\alpha(b)$ as well as $\alpha\left(b^{\text {new }}\right)$. If $y_{k}=-1$, the proof is the same, but equation A.5 becomes $\sum_{i: y_{i}=y_{k}} \alpha(b)_{i}<$ $\sum_{i: y_{i}=y_{k}} \alpha\left(b^{\text {new }}\right)_{i}$. A similar contradiction can be reached if $b^{\text {new }}<b$. Thus, $B$ is a singleton and $b$ is unique.

A.2 Optimal Solution of Equation 2.5. KKT conditions applied to equation 2.5 correspond to the existence of a scalar $b$ and two nonnegative vectors $\lambda$ and $\mu$ such that

$$
\begin{aligned}
& \alpha_{i}-1+b y_{i}=\lambda_{i}-\mu_{i}, \\
& \alpha_{i} \lambda_{i}=0, \quad\left(C-\alpha_{i}\right) \mu_{i}=0, \quad i=1, \ldots, l .
\end{aligned}
$$


To show that the solution is given by equations 2.6 and 2.7 , all that we need to do is to show the existence of $\lambda$ and $\mu$ so that equation A. 6 holds. For the solution 2.6, when $C \geq C_{\lim }$, using $b$ defined in equation 2.7,

$$
\alpha_{i}-1+b y_{i}=0 \forall i
$$

so we can simply choose $\lambda=\mu=0$ so that equation A. 6 is satisfied.

If $C<C_{\text {lim }}$

$$
\alpha_{i}-1+b y_{i}= \begin{cases}0 & \text { if } y_{i}=1 \\ \frac{C l}{l_{1}}-2 \leq 0 & \text { if } y_{i}=-1\end{cases}
$$

so equation A.6 also holds. Therefore, equation 2.6 gives an optimal solution for equation 2.5 .

Let us now analyze properties of the classifier function $f$ associated with equation 2.5. Note using equation 2.7 that $b>0$. For $x \neq x_{i}, \exp (-\| x-$ $\left.x_{i} \|^{2} / \sigma^{2}\right) \rightarrow 0$ as $\sigma^{2} \rightarrow 0$. Therefore, for such $x$, the classifier function corresponding to equation 2.5 is given by $f(x)=b$. Since $b>0$, all points $x$ not in the training set are classified as class 1 regardless of the value of $C$. This, together with item 2 of assumption 2, implies that the number of LOO errors is equal to $l_{2}$.

For a training point $x_{i}$, we have $f\left(x_{i}\right) \rightarrow y_{i} \alpha_{i}+b$ as $\sigma^{2} \rightarrow 0$. Thus, after $\sigma^{2}$ is sufficiently small, all class 1 training points are classified correctly by $f$. For training points $x_{i}$ in class 2 , we can use equations 2.6 and 2.7 to show that (i) for $C>C_{\lim } / 2$, all of those points are classified correctly by $f$, and (ii) for $C \leq C_{\lim } / 2$, all of those points are classified incorrectly by $f$.

A.3 Proof of Theorem 1. To prove theorem 1, first we write down the primal form of equation 2.11:

$$
\begin{array}{ll}
\min _{\tilde{w}, \tilde{b}, \tilde{\xi}} & \frac{1}{2} \tilde{w}^{T} \tilde{w}+\tilde{C} \sum_{i=1}^{l} \tilde{\xi}_{i} \\
\text { subject to } & y_{i}\left(\tilde{w}^{T} \tilde{\phi}\left(x_{i}\right)+\tilde{b}\right) \geq 1-\tilde{\xi}_{i}, \\
& \tilde{\xi}_{i} \geq 0, i=1, \ldots, l,
\end{array}
$$

where $\tilde{\phi}(x) \equiv \sigma \phi(x) .{ }^{6}$ By defining $w \equiv \sigma \tilde{w}$, multiplying the objective function of equation A.7 by $\sigma^{2}$ and using equation 2.12, equation A.7 has exactly the same form as equation 1.1, so we can say,

$$
b=\tilde{b}, \xi=\tilde{\xi} \text {, and } \tilde{w}^{T} \tilde{\phi}(x)+\tilde{b}=w^{T} \phi(x)+b .
$$

\footnotetext{
${ }^{6}$ It should be pointed out that equation 2.11 is not directly the dual of equation A.7. The dual of equation A.7 reduces to equation 2.11 when the $y^{T} \tilde{\alpha}=0$ constraint is used.
} 
A difficulty in proving this theorem is that the solution of equation A.7 is an element of a vector space that is different from that of a solution of equation 2.14. Hence, to build the relation as $\sigma^{2} \rightarrow \infty$, we will consider their duals using lemma 2 .

Assume $\tilde{\alpha}\left(\sigma^{2}\right)$ is the solution of equation 2.11 under a given $\tilde{C}$. It is in a bounded region for all $\sigma^{2}>0$ so there is a convergent sequence $\tilde{\alpha}\left(\sigma_{k}^{2}\right) \rightarrow \tilde{\alpha}$ as $\sigma_{k}^{2} \rightarrow \infty$. We can apply lemma 2 , as now the $i j$ component of the Hessian of equation 2.11 is a function of $\sigma^{2}$ :

$$
y_{i} y_{j} \tilde{K}_{i j}=\sigma^{2} y_{i} y_{j}\left(e^{-\left\|x_{i}-x_{j}\right\| /\left(2 \sigma^{2}\right)}-1+\frac{\left\|x_{i}\right\|^{2}}{2 \sigma^{2}}+\frac{\left\|x_{j}\right\|^{2}}{2 \sigma^{2}}\right) \text {, }
$$

with the limit $y_{i} y_{j} x_{i}^{T} x_{j}$ as $\sigma^{2} \rightarrow \infty$. Therefore, $\tilde{\alpha}\left(\sigma_{k}^{2}\right)$ converges to an optimal solution $\tilde{\alpha}$ of equation 2.13 .

We denote that $\tilde{w}\left(\sigma^{2}\right)$ and $\tilde{w}$ are unique optimal solutions of equations A.7 and 2.14, respectively. Then, for any such convergent sequence $\left\{\tilde{\alpha}\left(\sigma_{k}^{2}\right)\right\}_{k=1}^{\infty}$ using $y^{T} \tilde{\alpha}\left(\sigma_{k}^{2}\right)=0$, we have that for any $x$,

$$
\begin{aligned}
\lim _{\sigma_{k}^{2} \rightarrow \infty} \tilde{w}\left(\sigma_{k}^{2}\right)^{T} \tilde{\phi}(x) \\
\quad=\lim _{\sigma_{k}^{2} \rightarrow \infty} \sum_{i=1}^{l} y_{i} \tilde{\alpha}\left(\sigma_{k}^{2}\right)_{i} \tilde{\phi}\left(x_{i}\right)^{T} \tilde{\phi}(x) \\
=\lim _{\sigma_{k}^{2} \rightarrow \infty} \sum_{i=1}^{l} y_{i} \tilde{\alpha}\left(\sigma_{k}^{2}\right)_{i}\left(\sigma_{k}^{2}-\left\|x_{i}\right\|^{2} / 2+x_{i}^{T} x-\|x\|^{2} / 2\right) \\
=\lim _{\sigma_{k}^{2} \rightarrow \infty} \sum_{i=1}^{l} y_{i} \tilde{\alpha}\left(\sigma_{k}^{2}\right)_{i}\left(-\left\|x_{i}\right\|^{2} / 2+x_{i}^{T} x\right) \\
=\sum_{i=1}^{l} y_{i} \tilde{\alpha}_{i} x_{i}^{T} x+d(\tilde{\alpha})=\tilde{w}^{T} x+d(\tilde{\alpha}),
\end{aligned}
$$

where equation A.9 follows from equation 2.8 and $y^{T} \tilde{\alpha}\left(\sigma_{k}^{2}\right)=0$ and $d(\tilde{\alpha}) \equiv$ $-\sum_{i=1}^{l} y_{i} \tilde{\alpha}\left\|x_{i}\right\|^{2} / 2$. By a similar way, we can prove

$$
\begin{aligned}
\lim _{\sigma_{k}^{2} \rightarrow \infty} \tilde{w}\left(\sigma_{k}^{2}\right)^{T} \tilde{w}\left(\sigma_{k}^{2}\right) \\
=\lim _{\sigma_{k}^{2} \rightarrow \infty} \sum_{i=1}^{l} \sum_{j=1}^{l} \tilde{\alpha}\left(\sigma_{k}^{2}\right)_{i} \tilde{\alpha}\left(\sigma_{k}^{2}\right)_{j} y_{i} y_{j} \tilde{\phi}\left(x_{i}\right)^{T} \tilde{\phi}\left(x_{j}\right) \\
=\sum_{i=1}^{l} \sum_{j=1}^{l} \tilde{\alpha}\left(\sigma_{k}^{2}\right)_{i} \tilde{\alpha}\left(\sigma_{k}^{2}\right)_{j} y_{i} y_{j} x_{i}^{T} x_{j}=\tilde{w}^{T} \tilde{w} .
\end{aligned}
$$


Note that equation A.11 follows from the discussion between equations 2.8 and 2.11. The last equality is via $\tilde{w}=\sum_{i=1}^{l} \tilde{\alpha}_{o} x_{i}$, as $\tilde{w}$ is the optimal solution of equation 2.11 .

Next we consider that $(\tilde{w}, \tilde{b})$ is the unique optimal solution of equation 2.14. The constraints of equation A.7 imply that

$$
\begin{aligned}
\max _{y_{i}=1} & \left\{1-\tilde{w}\left(\sigma^{2}\right)^{T} \tilde{\phi}\left(x_{i}\right)-\tilde{\xi}\left(\sigma^{2}\right)_{i}\right\} \\
& \leq \tilde{b}\left(\sigma^{2}\right) \leq \max _{y_{i}=-1}\left\{-1-\tilde{w}\left(\sigma^{2}\right)^{T} \tilde{\phi}\left(x_{i}\right)+\tilde{\xi}\left(\sigma^{2}\right)_{i}\right\} .
\end{aligned}
$$

Note that the primal-dual optimality condition implies

$$
0 \leq \tilde{\xi}\left(\sigma^{2}\right)_{i} \leq \sum_{i=1}^{l} \tilde{\xi}\left(\sigma^{2}\right)_{i} \leq \frac{e^{T} \tilde{\alpha}\left(\sigma^{2}\right)}{\tilde{C}} \leq l .
$$

With equation A.9 and the assumption $l_{1} \geq 1$ and $l_{2} \geq 1$, after $\sigma^{2}$ is large enough, $\tilde{b}\left(\sigma^{2}\right)$ is in a bounded region. When $\left(\tilde{w}\left(\sigma^{2}\right), \tilde{b}\left(\sigma^{2}\right)\right)$ is optimal for equation A.7, the optimal $\tilde{\xi}\left(\sigma^{2}\right)$ is

$$
\tilde{\xi}\left(\sigma^{2}\right)_{i} \equiv \max \left(0,1-y_{i}\left(\tilde{w}\left(\sigma^{2}\right)^{T} \tilde{\phi}\left(x_{i}\right)+\tilde{b}\left(\sigma^{2}\right)\right)\right) .
$$

For any convergent sequence $\tilde{b}\left(\sigma_{k}^{2}\right) \rightarrow b^{*}$ with $\sigma_{k}^{2} \rightarrow \infty$, we can further have a subsequence such that $\left\{\tilde{\alpha}\left(\sigma_{k}^{2}\right)\right\}$ converges. Thus, we can consider any such sequence with both properties. Then equation A.10 implies

$$
\tilde{\xi}\left(\sigma_{k}^{2}\right)_{i} \rightarrow \xi_{i}^{*}=\max \left(0,1-y_{i}\left(\tilde{w}^{T} x_{i}+d(\tilde{\alpha})+b^{*}\right)\right) .
$$

Hence, $\left(\tilde{w}, b^{*}+d(\tilde{\alpha}), \xi^{*}\right)$ is feasible for equation 2.14. By defining

$$
\bar{\xi}\left(\sigma_{k}^{2}\right)_{i} \equiv \max \left(0,1-y_{i}\left(\tilde{w}\left(\sigma_{k}^{2}\right)^{T} \tilde{\phi}\left(x_{i}\right)-d(\tilde{\alpha})+\tilde{b}\right)\right),
$$

$\left(\tilde{w}\left(\sigma_{k}^{2}\right), \tilde{b}-d(\tilde{\alpha}), \bar{\xi}\left(\sigma_{k}^{2}\right)\right)$ is feasible for equation A.7. In addition, using equation A.10,

$$
\tilde{\xi} \equiv \lim _{\sigma_{k}^{2} \rightarrow \infty} \bar{\xi}\left(\sigma_{k}^{2}\right)=\max \left(0,1-y_{i}\left(\tilde{w}^{T} x_{i}+\tilde{b}\right)\right),
$$

so $(\tilde{w}, \tilde{b}, \tilde{\xi})$ is optimal for equation 2.14 . Thus,

$$
\begin{gathered}
\frac{1}{2} \tilde{w}^{T} \tilde{w}+\tilde{C} \sum_{i=1}^{l} \tilde{\xi}_{i} \leq \frac{1}{2} \tilde{w}^{T} \tilde{w}+\tilde{C} \sum_{i=1}^{l} \xi_{i}^{*}, \quad \text { and } \\
\frac{1}{2} \tilde{w}\left(\sigma_{k}^{2}\right)^{T} \tilde{w}\left(\sigma_{k}^{2}\right)+\tilde{C} \sum_{i=1}^{l} \tilde{\xi}\left(\sigma_{k}^{2}\right)_{i} \leq \frac{1}{2} \tilde{w}\left(\sigma_{k}^{2}\right)^{T} \tilde{w}\left(\sigma_{k}^{2}\right)+\tilde{C} \sum_{i=1}^{l} \bar{\xi}\left(\sigma_{k}^{2}\right)_{i} .
\end{gathered}
$$


With equations A.11, A.12, and A.13, taking the limit A.14 becomes

$$
\frac{1}{2} \tilde{w}^{T} \tilde{w}+\tilde{C} \sum_{i=1}^{l} \xi_{i}^{*} \leq \frac{1}{2} \tilde{w}^{T} \tilde{w}+\tilde{C} \sum_{i=1}^{l} \tilde{\xi}_{i}
$$

Therefore, we have that $\left(\tilde{w}, b^{*}+d(\tilde{\alpha}), \xi^{*}\right)$ is optimal for equation 2.14 . Since $\tilde{b}$ is unique by assumption,

$$
b^{*}+d(\tilde{\alpha})=\tilde{b} .
$$

Now we are ready to prove the main result, equation 2.15. If it is wrong, there is $\epsilon>0$ and a sequence $\left\{\tilde{w}\left(\sigma_{k}^{2}\right)\right\}$ with $\sigma_{k}^{2} \rightarrow \infty$ such that

$$
\left|\tilde{w}\left(\sigma_{k}^{2}\right)^{T} \tilde{\phi}(x)+b\left(\sigma_{k}^{2}\right)-\tilde{w}^{T} x-\tilde{b}\right| \geq \epsilon, \forall k .
$$

Since we can find an infinite subset $K$ such that $\lim _{k \in K, \sigma_{k}^{2} \rightarrow \infty} \tilde{b}\left(\sigma_{k}^{2}\right)=b^{*}$ and equation A.10 holds, with $b\left(\sigma^{2}\right)=\tilde{b}\left(\sigma^{2}\right)$ from equation A.8, the above analysis (equations A.10 and A.15) shows that

$$
\begin{aligned}
\lim _{k \in K, \sigma_{k}^{2} \rightarrow \infty} w\left(\sigma_{k}^{2}\right)^{T} \phi(x)+b\left(\sigma_{k}^{2}\right) & =\tilde{w}^{T} x+d(\tilde{\alpha})+\tilde{b}-d(\tilde{\alpha}) \\
& =\tilde{w}^{T} x+\tilde{b} .
\end{aligned}
$$

This contradicts equation A.16 so equation 2.15 is valid.

Therefore, if $\tilde{w}^{T} x+\tilde{b} \neq 0$, after $\sigma^{2}$ is sufficiently large,

$$
\operatorname{sgn}\left(w\left(\sigma^{2}\right)^{T} \phi(x)+b\left(\sigma^{2}\right)\right)=\operatorname{sgn}\left(\tilde{w}^{T} x+\tilde{b}\right) .
$$

A.4 Proof of Theorem 2. Let $\alpha^{1}$ be a feasible vector of equation 1.2 for $C=C_{1}$ and $\alpha^{2}$ be a feasible vector of equation 1.2 for $C=C_{2}$. We say that $\alpha^{1}$ and $\alpha^{2}$ are on the same face if the following hold: (i) $\{i \mid 0<$ $\left.\alpha_{i}^{1}<C_{1}\right\}=\left\{i \mid 0<\alpha_{i}^{2}<C_{2}\right\}$; (ii) $\left\{i \mid \alpha_{i}^{1}=C_{1}\right\}=\left\{i \mid \alpha_{i}^{2}=C_{2}\right\}$ and (iii) $\left\{i \mid \alpha_{i}^{1}=0\right\}=\left\{i \mid \alpha_{i}^{2}=0\right\}$. To prove theorem 2, we need the following result:

Lemma 4. If $C_{1}<C_{2}$ and their corresponding duals have optimal solutions at the same face, then for any $C_{1} \leq C \leq C_{2}$, there is at least one optimal solution at the same face. Furthermore, there are optimal solutions $\alpha$ and $b$ of equation 1.2, which form linear functions of $C$ in $\left[C_{1}, C_{2}\right]$.

Proof of Lemma 4. If $\alpha^{1}$ and $\alpha^{2}$ are optimal solutions at the same face corresponding to $C_{1}$ and $C_{2}$, then they satisfy the following KKT conditions, 
respectively:

$$
\begin{array}{lll}
Q \alpha^{1}-e+b^{1} y=\lambda^{1}-\mu^{1}, & \lambda_{i}^{1} \alpha_{i}^{1}=0, & \left(C^{1}-\alpha_{i}^{1}\right) \mu_{i}^{1}=0, \\
Q \alpha^{2}-e+b^{2} y=\lambda^{2}-\mu^{2}, & \lambda_{i}^{2} \alpha_{i}^{2}=0, & \left(C^{2}-\alpha_{i}^{2}\right) \mu_{i}^{2}=0 .
\end{array}
$$

Since they are at the same face,

$$
\begin{aligned}
& \lambda_{i}^{2} \alpha_{i}^{1}=0, \quad \lambda_{i}^{1} \alpha_{i}^{2}=0, \\
& \left(C^{1}-\alpha_{i}^{1}\right) \mu_{i}^{2}=0, \quad\left(C^{2}-\alpha_{i}^{2}\right) \mu_{i}^{1}=0 .
\end{aligned}
$$

As $C_{1} \leq C \leq C_{2}$, we can have $0 \leq \tau \leq 1$ such that

$$
C=\tau C_{1}+(1-\tau) C_{2} .
$$

Let

$$
\begin{array}{ll}
\alpha \equiv \tau \alpha^{1}+(1-\tau) \alpha^{2}, & \lambda \equiv \tau \lambda^{1}+(1-\tau) \lambda^{2}, \\
\mu \equiv \tau \mu^{1}+(1-\tau) \mu^{2}, & b \equiv \tau b^{1}+(1-\tau) b^{2} .
\end{array}
$$

Then $\alpha, \lambda, \mu, b$ satisfy the KKT condition at $C$ :

$$
\begin{aligned}
& Q \alpha-e+b y=\lambda-\mu, \quad \lambda_{i} \alpha_{i}=0, \quad\left(C-\alpha_{i}\right) \mu_{i}=0, \\
& 0 \leq \alpha_{i} \leq C, \quad \lambda_{i} \geq 0, \quad \mu_{i} \geq 0, \quad y^{T} \alpha=0 .
\end{aligned}
$$

Using equation A.18,

$$
\tau=\frac{C-C_{2}}{C_{1}-C_{2}} .
$$

Putting it into equation A.19, $\alpha$ and $b$ are linear functions of $C$ where $C \in$ $\left[C_{1}, C_{2}\right]$. This proves the lemma.

Let us now prove theorem 2 .

Proof of Theorem 3. As we already mentioned, if the points of the two classes are linearly separable in $x$ space, then the proof of the result is straightforward. So let us give a proof only for the case of linearly nonseparable points. Since the number of faces is finite, by lemma 4 there exists a $C^{*}$ such that for $C \geq C^{*}$, there are optimal solutions at the same face. For the rest of the proof, let us consider only optimal solutions on a single face.

For any $C_{1}>C^{*}$, lemma 4 implies that there are optimal solutions $\alpha$ and $b$, which form linear functions of $C$ in the interval $\left[C^{*}, C_{1}\right]$. Since

$$
\sum_{i=1}^{l} \xi_{i}=\sum_{i: \alpha_{i}=C}-\left[(Q \alpha)_{i}-1+b y_{i}\right]
$$


$\sum_{i=1}^{l} \xi_{i}$ is a linear function of $C$ in this interval and can be represented as

$$
\sum_{i=1}^{l} \xi_{i}=A C+B
$$

where $A$ and $B$ are constants. If we consider another $C_{2}>C_{1}, \sum_{i=1}^{l} \xi_{i}$ is also a linear function of $C$ in $\left[C^{*}, C_{2}\right]$. For each $C$, the optimal $\frac{1}{2} w^{T} w$ as well as $\sum_{i=1}^{l} \xi_{i}$ are unique. Thus, the two linear functions have the same values at more than two points, so they are indeed identical. Therefore, equation A.21 holds for any $C \geq C^{*}$.

Since $\sum_{i=1}^{l} \xi_{i}$ is a decreasing function of $C$ (e.g., using techniques similar to Chang and $\operatorname{Lin}(2001 \mathrm{~b}$, lemma 4$)), A \leq 0$. However, $A$ cannot be negative, as otherwise $\sum_{i=1}^{l} \xi_{i}$ goes to $-\infty$ as $C$ increases. Hence, $A=0$, and so $\sum_{i=1}^{l} \xi_{i}$ is a constant for $C \geq C^{*}$.

If $\left(w^{1}, b^{1}, \xi^{1}\right)$ and $\left(w^{2}, b^{2}, \xi^{2}\right)$ are optimal solutions at $C=C_{1}$ and $C_{2}$, respectively, then

$$
\frac{1}{2}\left(w^{1}\right)^{T} w^{1}+C_{1} \sum_{i=1}^{l} \xi_{i}^{1} \leq \frac{1}{2}\left(w^{2}\right)^{T} w^{2}+C_{1} \sum_{i=1}^{l} \xi_{i}^{2}
$$

and

$$
\frac{1}{2}\left(w^{2}\right)^{T} w^{2}+C_{2} \sum_{i=1}^{l} \xi_{i}^{2} \leq \frac{1}{2}\left(w^{1}\right)^{T} w^{1}+C_{2} \sum_{i=1}^{l} \xi_{i}^{1}
$$

imply that $\left(w^{1}\right)^{T} w^{1}=\left(w^{2}\right)^{T} w^{2}$. That is, $\alpha^{T} Q \alpha$ is a constant for $C \geq C^{*}$.

Therefore $\left(w^{2}, b^{2}, \xi^{2}\right)$ is also feasible and optimal when $C=C_{1}$. Since the solution of $w$ is unique (e.g., Lin, 2001, lemma 1), $w^{1}=w^{2}$.

If $F=\left\{i \mid 0<\alpha_{i}<C\right\} \neq \emptyset$, there is $x_{i}$ such that $w^{T} x_{i}+b=y_{i}$. Hence $b^{1}=b^{2}$, and so the decision functions as well as the LOO rates are the same for $C \geq C^{*}$.

On the other hand, if $F=\emptyset$ and we denote $\alpha(C)$ the solution of equation 1.2 at a given $C$, then $\alpha(C)=\left(C / C^{*}\right) \alpha\left(C^{*}\right)$ for all $C \geq C^{*}$. As $w^{T} w=$ $\alpha^{T} Q \alpha$ becomes a constant, we have $w=0$ after $C \geq C^{*}$. However, since $F=\emptyset$, the optimal $b$ might not be unique under the same $C$. For any one of such $b,(w, b)$ is optimal for equation 1.2 for all $C \geq C^{*}$.

Finally, since $w^{T} w$ becomes a constant, for $C \geq C^{*}$, the solution of equation 1.2 is also a solution of

$$
\begin{aligned}
\min _{w, b, \xi} & \sum_{i=1}^{l} \xi_{i} \\
\text { subject to } & y_{i}\left(w^{T} x_{i}+b\right) \geq 1-\xi_{i}, \\
& \xi_{i} \geq 0, i=1, \ldots, l .
\end{aligned}
$$

This completes the proof of theorem 2 . 


\section{Acknowledgments}

C.-J. L. was partially supported by the National Science Council of Taiwan, grant NSC 90-2213-E-002-111.

\section{References}

Blake, C. L., \& Merz, C. J. (1998). UCI repository of machine learning databases (Technical Rep.) Irvine, CA: University of California, Department of Information and Computer Science. Available on-line at: http://www.ics.uci. edu/ mlearn/MLRepository.html.

Chang, C.-C., \& Lin, C.-J. (2001a). LIBSVM: A library for support vector machines. Software Available on-line at: http://www.csie.ntu.edu.tw/ cjlin/libsvm.

Chang, C.-C., \& Lin, C.-J. (2001b). Training v-support vector classifiers: Theory and algorithms. Neural Computation, 13(9), 2119-2147.

Joachims, T. (2000). Estimating the generalization performance of a SVM efficiently. In Proceedings of the International Conference on Machine Learning. San Mateo, CA: Morgan Kaufmann.

Lee, J.-H. (2001). Model selection of the bounded SVM formulation using the RBF kernel. Master's thesis, National Taiwan University.

Lin, C.-J. (2001). Formulations of support vector machines: A note from an optimization point of view. Neural Computation, 13(2), 307-317.

Micchelli, C. A. (1986). Interpolation of scattered data: Distance matrices and conditionally positive definite functions. Constructive Approximation, 2, 11-22.

Platt, J. C. (1998). Fast training of support vector machines using sequential minimal optimization. In B. Schölkopf, C. J. C. Burges, \& A. J. Smola (Eds.), Advances in kernel methods-Support vector learning. Cambridge, MA: MIT Press.

Rätsch, G. (1999). Benchmark data sets. Available on-line at:http://ida.first.gmd. de/ raetsch/data/benchmarks.htm.

Vapnik, V. (1998). Statistical learning theory. New York: Wiley.

Vapnik, V., \& Chapelle, O. (2000). Bounds on error expectation for support vector machines. Neural Computation, 12(9), 2013-2036.

Received June 25, 2002; accepted December 5, 2002. 